\title{
Survey of two-time physics
}

\author{
Itzhak Bars \\ CIT-USC Center for Theoretical Physics and Department of Physics and Astronomy, University \\ of Southern California, Los Angeles, CA 90089-2535, USA
}

Received 16 October 2000

Published 1 August 2001

Online at stacks.iop.org/CQG/18/3113

\begin{abstract}
Two-time physics (2T) is a general reformulation of one-time physics (1T) that displays previously unnoticed hidden symmetries in $1 \mathrm{~T}$ dynamical systems and establishes previously unknown duality-type relations among them. This may play a role in displaying the symmetries and constructing the dynamics of little understood systems, such as M-theory. 2T-physics describes various 1T dynamical systems as different $d$-dimensional 'holographic' views of the same $2 \mathrm{~T}$ system in $d+2$ dimensions. The 'holography' is due to gauge symmetries that tend to reduce the number of effective dimensions. Different $1 \mathrm{~T}$ evolutions (i.e. different Hamiltonians) emerge from the same 2T-theory when gauge fixing is done with different embeddings of $d$ dimensions inside $d+2$ dimensions. Thus, in the $2 \mathrm{~T}$ setting, the distinguished $1 \mathrm{~T}$ which we call 'time' is a gaugedependent concept. The $2 \mathrm{~T}$-action also has a global $S O(d, 2)$ symmetry in flat spacetime, or a more general $d+2$ symmetry in curved spacetime, under which all dimensions are on an equal footing. This symmetry is observable in many 1T-systems, but it remained unknown until discovered in the $2 \mathrm{~T}$ formalism. The symmetry takes various nonlinear (hidden) forms in the 1T-systems, and it is realized in the same irreducible unitary representation (the same Casimir eigenvalues) in their quantum Hilbert spaces. 2T-physics has mainly been developed in the context of particles, including spin and supersymmetry, but some advances have also been made with strings and $p$-branes, and insights for M-theory have already emerged. In the case of particles, there exists a general worldline formulation with background fields, as well as a field theory formulation, both described in terms of fields that depend on $d+2$ coordinates. All 1T particle interactions with Yang-Mills, gravitational and other fields are included in the $d+2$ reformulation. In particular, the standard model of particle physics can be regarded as a gauge-fixed form of a 2T-theory in $4+2$ dimensions. These facts already provide evidence for a new type of higher-dimensional unification.
\end{abstract}

PACS numbers: 1210,1125 


\section{Introduction}

Although two-time physics [1-11] is currently best understood in simple everyday physics, it originally developed from hints about two-timelike dimensions in the mathematical structure of M-theory. In particular, the first hint came from the 11-dimensional extended superalgebra, including the 2-brane and 5-brane charges which has the form [12]

$$
\left\{Q_{\alpha}, Q_{\beta}\right\}=\gamma_{\alpha \beta}^{\mu} P_{\mu}+\gamma_{\alpha \beta}^{\mu \nu} Z_{\mu \nu}+\gamma_{\alpha \beta}^{\mu_{1} \cdots \mu_{5}} Z_{\mu_{1} \cdots \mu_{5}} .
$$

It was noted that this structure provides a model-independent signal for 12 dimensions in Mtheory with $(10,2)$ signature [13], since the 32 supercharges may be viewed as a Weyl spinor in 12 dimensions and the 528 bosonic charges may be viewed as a 2-form plus a self-dual 6-form in 12 dimensions,

$$
P_{\mu} \oplus Z_{\mu \nu}=Z_{M N}, \quad Z_{\mu_{1} \cdots \mu_{5}}=Z_{M_{1} \cdots M_{6}}^{+} .
$$

This observation has been generalized in several directions, including S-theory [14] and several of its applications that lend further support to this view. Taking into account various dualities, 13 dimensions with $(11,2)$ signature appeared more appealing because in that framework S-theory can unify type-IIA and type-IIB supersymmetric systems in 10 dimensions.

There are several other observations that support two timelike dimensions. These include the brane scan [15], $N=2$ superstrings [16], F-theory [17], U-theory [18], the hints for a 12D super-Yang-Mills or supergravity theory [19], the AdS-CFT correspondence [20], etc.

The question is whether these hints imply that two timelike dimensions exist? Can they be made manifest in a formulation of the fundamental theory including explicitly two timelike dimensions with the associated symmetries? Historically, in previous failed attempts for more timelike dimensions, some formidable obstacles to overcome included causality and unitarity, the latter being due to ghosts (negative norm states) created by extra timelike dimensions. Because of these fundamental problems extra timelike dimensions could not be hidden away by treating them naively like extra spacelike dimensions and pretending that they are compactified in little circles.

The answer to the fundamental problems could only be a new gauge symmetry that removes the ghosts and establishes both unitarity and causality. Two-time physics introduced a new symplectic gauge symmetry which indeed removes all ghosts, establishes unitarity and causality, and plays a role analogous to duality ${ }^{1}$. Two-time physics is verified in everyday physics that has already been understood. Besides bringing the new $(d+2)$-dimensional insights to well known physics, it is hoped that the formalism would be helpful in the formulation of fundamental physics that remains to be understood.

The gauge symmetry that plays a fundamental role in two-time physics historically evolved from attempts to give a dynamical description of S-theory. In that vein, new gauge symmetry concepts were developed in [27] for constrained multi-particle or particle-string systems which require two-timelike dimensions for a consistent formulation. Eventually these efforts led to the formulation of the gauge symmetry for a single particle as given in [1].

The gauge symmetry is very natural and could have been explored independent of $\mathrm{M}$ theory, S-theory or other motivations. It arises as follows: in the first-order formulation of any theory the action has the form $S=\int[\dot{X} \cdot P-H(X, P)]$. Up to an irrelevant total derivative the first term can be rewritten as $\int \frac{1}{2}[\dot{X} \cdot P-\dot{P} \cdot X]=\int \frac{1}{2} \dot{X}_{i} \cdot X_{j} \epsilon^{i j}$, where $\left(X^{M}, P^{M}\right)=X_{i}^{M}$ is the $\operatorname{Sp}(2, R)$ doublet in phase space. This shows that the first term in the action has a

1 It may be significant that the S, T, U dualities in M-theory or the Seiberg-Witten dualities in super-Yang-Mills theory are also (discrete) gauge symmetries of a symplectic nature that mix canonically conjugate quantities (such as electric-magnetic $p$-brane charges, or windings in space and Kaluza-Klein momenta, etc). 
global $\operatorname{Sp}(2, R)$ symmetry. Furthermore, the same $\operatorname{Sp}(2, R)$ appears as an automorphism symmetry in the quantum relations $\left[X_{i}^{M}, X_{j}^{N}\right]=\mathrm{i} \epsilon_{i j} \eta^{M N}$. This global symmetry is present in every quantum relation of generalized coordinates and in every first term of the action for any dynamical system. A fundamental question is: what are the systems for which there is a local $\operatorname{Sp}(2, R)$ symmetry, not only in the first term, but in the full action? Also, if such a system exists, what are some interesting generalizations of this local symmetry? This fundamental question may be taken as the basic starting point for two-time physics. The two times and all other consequences (unification of 1T-systems, etc) follow directly from the gauge symmetry. In particular, two times (no less and no more!) is an outcome of the gauge symmetry, it is not put in by hand. Thus, two-time physics arises from a gauge principle.

In these lecture notes I will mainly emphasize the concepts of 2T-physics. I will briefly summarize some facts and results whose details are found in the literature ${ }^{2}$ [1-11].

\section{2T follows from gauge symmetry}

In the worldline formulation of particles the new gauge symmetry, and its generalizations, act on phase space. The first-class constraints associated with the gauge symmetry ensure unitarity (no ghosts). These constraints have non-trivial solutions only if the target spacetime has two timelike dimensions. Thus, the gauge symmetry demands that neither fewer nor more timelike dimensions are permitted in the description of a single point particle (the many-body theory described by the corresponding field theory also must have exactly two timelike dimensions).

For spinless particles the gauge symmetry is the $\operatorname{Sp}(2, R)$ that acts on position-momentum $\left(X^{M}, P^{M}\right)$ as a doublet. For spinning particles the gauge symmetry is $O S p(n \mid 2)$ which acts on super-phase space $\left(\psi_{a}^{M}, X^{M}, P^{M}\right)$ in its fundamental representation (where $a=1, \ldots, n$ ). With spacetime supersymmetry the gauge symmetry is enlarged with a new version of kappa supersymmetry in $d+2$ dimensions.

One consequence of the gauge symmetry in all cases is the requirement of two timelike dimensions in the target spacetime since otherwise the constraints have no non-trivial physical solutions. Thus the $2 \mathrm{~T}$ formulation is dictated by the gauge symmetry.

For example for spinless particles the $S p(2, R)$ constraints are $X^{2}=P^{2}=X \cdot P=0$. If the target spacetime signature in these dot products is Euclidean the only solution is $X^{M}=P^{M}=0$, and the theory is trivial. If it has one timelike direction, then $X^{M}$ and $P^{M}$ can only be lightlike vectors that are parallel, so there is no angular momentum, which is also trivial. If the signature has three timelike directions, then there are too many ghosts that cannot be removed by the $\operatorname{Sp}(2, R)$ gauge symmetry. Thus, only for the case with two timelike

2 After much work was done in 2T-physics, including background fields and field theory, I became aware of some results that were obtained independently by other authors [21-24]. The bulk of this literature originates with Dirac's formulation in 1936 of a six-dimensional field theory formalism whose goal was to understand the conformal $S O(4,2)$ symmetry in four-dimensional spacetime in a linear realization. Dirac and his followers were not aware of the underlying local $\operatorname{Sp}(2, R)$ symmetry which plays the fundamental role in 2T-physics. This local symmetry explains the origin of Dirac's field equations in six dimensions which he arrived at with a very different reasoning. Furthermore, one can understand that Dirac's (and other author's) path from six to four dimensions corresponds to one of the many possible gauge choices in 2T-physics. There was no awareness of the many paths of coming down from $d+2$ to $d$ dimensions which is related to the ability of making a variety of $\operatorname{Sp}(2, R)$ gauge choices. As a result, the older work missed one of the most important aspects of 2T-physics, namely the 'holographic' unification of different 1T-physical systems (different Hamiltonians) in a single 2T-action. It also missed the related different realizations of $S O(d, 2)$ that have different interpretations than conformal symmetry, thus reflecting the presence of the underlying $d+2$ dimensions. It was fortunate to have been unaware of this literature while 2T-physics developed, for it could have derailed the exploration of the new aspects revealed by 2T-physics. Of course, the past work did not address the $d+2$ formulation of more modern topics such as supersymmetry, $p$-branes, etc that have been developed in the context of 2T-physics. 
dimensions do the constraints have non-trivial solutions and the gauge symmetry removes all ghosts. In particular, out of the three gauge parameters in $\operatorname{Sp}(2, R)$, one is related to the familiar $\tau$-reparametrizations, while the other two have signatures such that they can remove one spacelike and one timelike dimension ${ }^{3}$.

For all other cases of gauge (super)symmetries mentioned above — or their generalizations to strings and branes - their content is as follows: there is just enough gauge symmetry to remove one timelike and one spacelike directions from all $S O(d, 2)$ vectors and also remove half of the $S O(d, 2)$ spinor components to arrive at $S O(d-1,1)$ spinors. Further generalizations of the gauge symmetries (see, e.g., the toy M-model in section 7.3) also remove ghost components from $S O(d, 2)$ tensors (such as antisymmetric tensors associated with branes).

\section{Unification of 1T-systems, 'holography'}

The simplest action with an $\operatorname{Sp}(2, R)$ gauge symmetry is [1]

$$
L=\dot{X}_{1} \cdot X_{2}-\frac{1}{2} A^{i j} X_{i} \cdot X_{j}
$$

where $X_{i}^{M}=\left(X^{M}, P^{M}\right)$ is the $\operatorname{Sp}(2, R)$ doublet, and $A^{i j}$ is the $\operatorname{Sp}(2, R)$ gauge potential. The equation of motion of the gauge potential leads to the constraints, hence the target spacetime must have signature $(d, 2)$ as an outcome of the $\operatorname{Sp}(2, R)$ gauge symmetry.

This system can be embellished with the addition of background fields [10] (Yang-Mills, gravity, others), spinning degrees of freedom [3], spacetime supersymmetry [5-9], and can be generalized to strings and $p$-branes [4]. For each generalization the $\operatorname{Sp}(2, R)$ gauge symmetry is enriched with a modified gauge symmetry.

A given 2T-physics system defined by an action in $(d, 2)$ dimensions $X^{M}$, displays a unification of a class of one-time dynamical systems in $(d-1,1)$ dimensions $x^{\mu}$. That is, a given 2T-action modulo gauge symmetries, is equivalent to many $1 \mathrm{~T}$ actions with different $1 \mathrm{~T}$ dynamics. The extra one-spacelike and one-timelike dimensions $(1,1)$ are interchangeable with gauge degrees of freedom. The different embeddings of $d$ dimensions $x^{\mu}$ inside $d+2$ dimensions $X^{M}$ produce different dynamics (different Hamiltonians) in the chosen $d$ dimensions. The dynamical systems obtained in this way belong to a class defined by a given $2 \mathrm{~T}$-action. Changing the $2 \mathrm{~T}$-action (e.g. changing background fields, etc) changes the class. The gauge symmetry relates the different $d$-dimensional $1 \mathrm{~T}$ actions (in the same class) to each other in a way reminiscent of 'duality'.

One may say that various $1 \mathrm{~T}$ actions in $d$ dimensions are different 'holographic' descriptions of the same $2 \mathrm{~T}$ system in $d+2$ dimensions. There is an equivalence between a family of different 'dynamics' and a family of different 'holographic' views. The simplest and most symmetric view is the non-holographic $(d+2)$-dimensional description.

The essential ideas of $(d+2)$-dimensional unification can already be understood in the simplest case. For the action (3) some members of the class of 1T spinless particle systems is given in figure 1. The action (or equations of motion) of those 1T dynamical systems emerge by gauge fixing this $2 \mathrm{~T}$ Lagrangian (or its equations of motion).

- Massless relativistic particle: $X^{+\prime}(\tau)=1, P^{+\prime}(\tau)=0$.

3 If one considers gauging $S p(2 n)$, instead of $S p(2)$, then one finds that the system describes $n$ particles with $2 n$ times in $(d+2 n-2,2 n)$ dimensions [26]. After partial gauge fixing and solving a subset of the $S p(2 n)$ constraints such a system reduces to the $n$-time $n$-particle theories in $(d+n-2, n)$ dimensions discussed in [27]. Fully solving all constraints one obtains $n$ particles each in $(d-1,1)$ dimensions, but each using a different timelike coordinate as embedded in the higher dimensions. So, to describe a single particle (and the associated field theory, i.e. a 1T many-body system) we can only have local $S p(2)$ and only two times. 


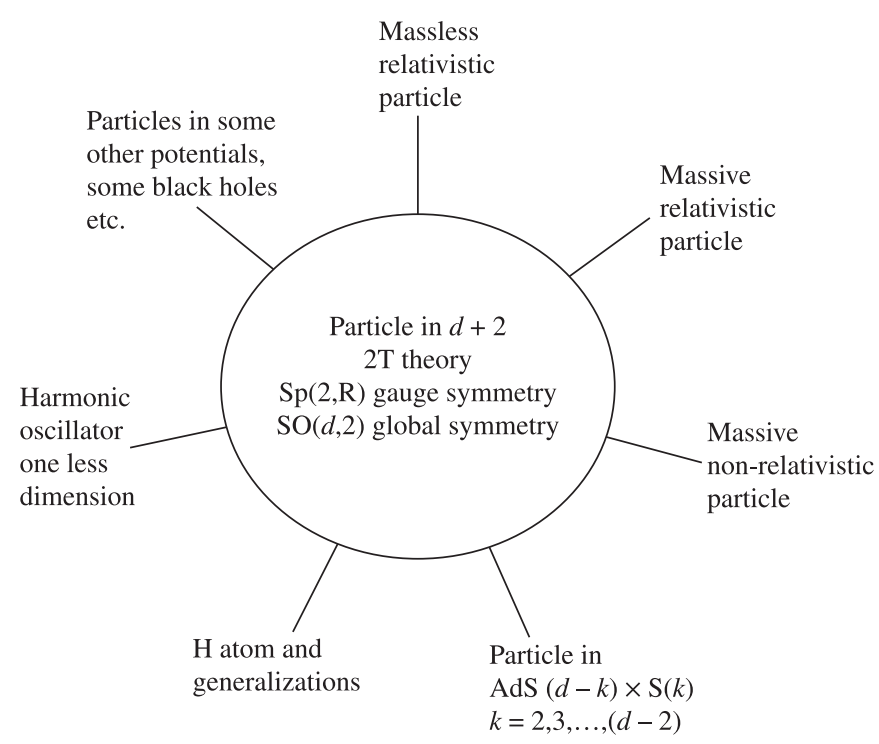

Figure 1.

- Massive non-relativistic particle: $P^{+1}(\tau)=m, P^{0}(\tau)=0$.

- Massive relativistic particle: $P^{+\prime}(\tau)=m, P^{0}(\tau)=0$.

- Particle on $A d S_{d}: X^{d-1}(\tau)=1, P^{+\prime}(\tau)=0$,

and so on for the $\mathrm{H}$-atom, harmonic oscillator, etc noted in the figure, and more (see $[1,2]$ for details). In each case one timelike dimension and one spacelike dimension is eliminated from each vector $\left(X^{M}, P^{M}\right)$ by the gauge choice and the solution of the two constraints $X^{2}=X \cdot P=0$. The remaining gauge choice and constraint $P^{2}=0$ is associated with the $\tau$-reparametrization gauge symmetry, and it may be convenient to gauge fix it to complete the relation between $\tau$ and the 1T-dimension embedded in $(d, 2)$ target spacetime $X^{M}(\tau)$. The theory, as expressed by the remaining coordinates, is then forced to evolve as a function of the remaining 1T timelike dimension. The Hamiltonian (canonical conjugate to the $1 \mathrm{~T}$ dimension) that describes this time evolution takes different forms in each case in terms of the remaining canonical degrees of freedom. Such embeddings of 'time' as a curve inside $d+2$ dimensions can be done in an infinite number of ways, thereby producing a class of related $1 \mathrm{~T}$ dynamical systems from the same 2T-theory.

When the 2T Lagrangian is changed by including spin, supersymmetry, background fields (but always maintaining a local $S p(2)$ symmetry), the class of related 1T-systems changes accordingly. Thus, the class of unified 1T-systems is defined by the 2T-action.

It is a fact, by construction, that the 1T 'time' and its associated Hamiltonian are gaugedependent concepts in this setting, and all 1T-systems in the same class are gauge related to the same 2T-theory. They can all be gauge transformed to each other, and these gauge transformations are the analogues of 'duality'. These are concepts that physicists will take some time to become accustomed to, but they will do so with a little effort, because these facts are easily established in simple systems as well as generally.

The relations between $1 \mathrm{~T}$ theories become more understandable by concentrating on gauge invariants such as the $S O(d, 2)$ global symmetry generators

$$
L^{M N}=X_{i}^{M} X_{j}^{N} \epsilon^{i j}=X^{M} P^{N}-X^{N} P^{M} .
$$


In any 1T-system the $d$ canonical coordinates that remain after gauge fixing $\left(x^{\mu}, p^{\mu}\right)$ can be rewritten as functions of the gauge invariant $L^{M N}$. Therefore, in any fixed 1T-system all physical quantities $F(x, p)$ can be rewritten in terms of functions of the gauge-invariant operators $f\left(L^{M N}\right)$. Those same functions have a different expression in terms of the canonical variables in another fixed 1T-system $\tilde{F}\left(\tilde{x}^{\mu}, \tilde{p}^{\mu}\right)$. However, because of the gauge invariance of $f\left(L^{M N}\right)$ they are guaranteed to produce identical results $F(x, p)=f\left(L^{M N}\right)=\tilde{F}\left(\tilde{x}^{\mu}, \tilde{p}^{\mu}\right)$. This observation permits the establishment of many relations between the 1T-systems in the same class. As long as one computes the same functions of $f\left(L^{M N}\right)$ it does not matter in which 1T-system it is computed. This is the test of the dualities which establishes that indeed all of the 1T-systems in the same class are related to the same 2T-theory ${ }^{4}$. All of this is easily carried out in the classical theory. So one may use a convenient gauge-fixed 1T version to perform computations that apply in all other related 1T-systems (for example, solving equations of motion, etc). In the quantum theory, operator ordering in nonlinear functions can produce anomalies, and one needs to find the correct ordering in transforming from one 1T-system to another (see [1-3] for some examples of quantum ordering, in particular in the computation of the Casimir operator $\frac{1}{2} L^{M N} L_{M N}$ in different gauge-fixed systems).

\section{Global symmetry, one evidence for $d+2$ dimensions}

In flat spacetime, before gauge fixing, the 2T-action in (3) has a global $S O(d, 2)$ symmetry which is linearly realized on $d+2$ coordinates $X^{M}$ and is manifest in the action. This is the Lorentz symmetry in $d+2$ dimensions which treats all coordinates on an equal footing. This symmetry is gauge invariant (commutes with the $\operatorname{Sp}(2, R)$ gauge transformations) so its generators (4) are physical observables. By the gauge invariance of the action (3) and of the symmetry generators (4) the same symmetry is present in every $1 \mathrm{~T}$ action derived from the same 2T-action by gauge fixing. In each $1 \mathrm{~T}$ action (such as those that correspond to figure 1) this symmetry is nonlinearly realized in different ways on the fewer $d$ coordinates $x^{\mu}$ singled out by a fixed gauge.

When the system is in interaction with background fields (see the next section), the global $S O(d, 2)$ symmetry is modified to the Killing symmetries permitted by those fields. In the field-theoretic formulation (see below), in the presence of gravity, the $S O(d, 2)$ symmetry is elevated to general coordinate invariance and Yang-Mills-type gauge symmetries in $d+2$ dimensions. Whatever the target spacetime symmetry may be, it is also present (perhaps in a hidden form) in the 1T-systems derived from the 2T-action. This simply follows from the reasoning in the previous paragraph.

This reasoning in the 2T-physics formulation permitted for the first time the discovery of $S O(d, 2)$ symmetry in a variety of familiar systems in 'everyday physics' [1-3]. In one $1 \mathrm{~T}$ gauge, corresponding to the massless particle in figure 1, the hidden symmetry was very familiar to the conformal $S O(d, 2)$ symmetry in massless systems. In another $1 \mathrm{~T}$ gauge, corresponding to the $\mathrm{H}$-atom in figure 1 , the hidden symmetry was also understood a long time ago, as a dynamical symmetry of the usual $\mathrm{H}$-atom which describes all of its quantum levels as

4 Some intuition for what is going on may be helpful for readers that are familiar with canonical transformations, by noting that there is a relation between the local $\operatorname{Sp}(2, R)$ 'duality' transformations from one 1T-system to another 1T-system, and generalized canonical transformations that connect the same systems including transformations of the time coordinate and the Hamiltonian. While this observation may be helpful initially to digest the new insights for simple systems, this relation by itself is not helpful more generally in understanding other phenomena in 2T-physics (e.g. the global symmetry discussed in the next paragraph). Furthermore, generalized canonical transformations is not a well developed method in the context of spin, supersymmetry (with generalized local kappa), field theory, $p$-branes, M-theory, etc. Therefore, the gauge symmetry formalism of 2T-physics is a far more superior and useful language even for the simple cases, and is a more general method in the long run. 
a single irreducible representation of $S O(4,2)$ (although no one had noticed previously that it is the classical symmetry of the $\mathrm{H}$-atom action). For all other $1 \mathrm{~T}$ gauge choices in figure 1 the $S O(d, 2)$ symmetry of the classical action has been verified [1-3]. Of course, the general argument guarantees that the symmetry is present at the classical level in every imaginable gauge choice of 1T. However, except for the two cases mentioned above, for all other cases the presence of the $S O(d, 2)$ symmetry came as a surprise to physicists.

It is now possible to claim that the various forms of $S O(d, 2)$ symmetry, such as conformal symmetry or other forms, are nothing but an expression of the higher-dimensional $(d+2)$ nature of the system, since the symmetry is nothing but the same Lorentz symmetry of the unified 2T-action that treats all dimensions on the same footing. The gauge choice blurs the higherdimensional nature of the system, but the symmetry is there to assert the presence of the higher dimensions. Therefore, there is a sense in which there are $d+2$ dimensions even when we concentrate on the 1T description of the system. A similar argument applies when $S O(d, 2)$ is replaced by a Killing symmetry in the presence of background fields.

Thus, the establishment of the target spacetime hidden $S O(d, 2)$ or similar Killing symmetry in many familiar 1T actions at the classical level is one evidence for the presence and relevance of the higher dimensions. The extra one-timelike and one-spacelike dimensions appear to have a different physical role from the remaining $d$ dimensions in a given fixed gauge from the 'holographic' point of view of 1T-physics. However, one can claim that this is only one 'non-democratic' way of observing the full system. In a different gauge, a different set of 'holographic' $d$ dimensions becomes distinguished to describe the same $(d+2)$-dimensional system. So there is a point of view in which the $(1,1)$ gauge degrees of freedom and the $(d-1,1)$ dimensions distinguished by a gauge choice are really on the same footing as far as the $S O(d, 2)$ symmetry or the spacetime dimensionality of the system is concerned.

What happens at the quantum level? To express the symmetry correctly, it is necessary to order carefully the operators $\left(x^{\mu}, p^{\mu}\right)$ that appear in nonlinear expressions of the generators $L^{M N}$. This has been done successfully in a few of the cases given in figure 1, and then it was possible to show that the $S O(d, 2)$ symmetry is realized in a Hilbert space which corresponds to the same unitary representation of $S O(d, 2)$ (same Casimir eigenvalues) for 1T-systems derived from the same 2T-action [1-3]. The various 1 T quantum systems are distinguished from each other by diagonalizing different subsets of compatible operators (functions of $S O(d, 2)$ generators) including the distinguished 1T Hamiltonian, but always staying in the Hilbert space with the same eigenvalues of the $S O(d, 2)$ Casimir operators, thus the same unitary representation of $S O(d, 2)$. The transformation from a 1T-system to another one involves the unitary transformation from one set of compatible operators to another one, but this transformation (which is related to the $S p(2, R)$ gauge symmetry) commutes with the $S O(d, 2)$ Casimirs and hence does not change the $S O(d, 2)$ representation, it only changes the basis. In this quantum space the unification of the 1T-systems and their duality-like relationships (of the type mentioned in the previous section $\left.F(x, p)=\tilde{F}\left(\tilde{x}^{\mu}, \tilde{p}^{\mu}\right)=f\left(L^{M N}\right)\right)$ could be computed most directly by using group-theoretical techniques ${ }^{5}$.

\footnotetext{
5 Generally, quantum operator ordering may not be easy for generic gauge choices. Normally the system in a given gauge would not be correctly quantized until the symmetry generators close and have the same Casimir eigenvalues as any other gauge (see [1-3] for explicit cases). However, one may raise the question if there are gauges in which the correct ordering could never be resolved, thus having fundamental anomalies in that gauge. It is not presently known whether this may be the case for some $2 \mathrm{~T}$-actions, in particular with background fields. In any case, anomalies of global symmetries (as opposed to local symmetries) would not invalidate the 2T-theory; they would simply be a property of the theory similar to anomalies in global symmetries encountered in field theory (e.g. the Adler-Bardeen anomaly, conformal anomaly).
} 


\section{Background fields}

The simplest action without spin in (3) is generalized to

$$
S=\int \mathrm{d} \tau\left(\partial_{\tau} X^{M} P_{M}-\frac{1}{2} A^{i j} Q_{i j}(X, P)\right),
$$

where a more general function of phase space $Q_{i j}(X, P)$ replaces the previous expressions $Q_{11}=X^{2}, Q_{12}=X \cdot P$ and $Q_{22}=P^{2}$. It is shown in [10] that this action has local $\operatorname{Sp}(2, R)$ gauge symmetry provided $A^{i j}$ transforms like a standard gauge field and $Q_{i j}(X, P)$ is any function of phase space that satisfies the $\operatorname{Sp}(2, R)$ Lie algebra under Poisson brackets. From the equations of motion of $A^{i j}$ we deduce that the constraints $Q_{i j}(X, P)=0$ define the physical states.

A general approach for finding non-trivial models of $Q_{i j}(X, P)$ is to set-up a series expansion in powers of $P^{M}$ for each $Q_{i j}$ with coefficients that are arbitrary functions of $X^{M}$. These coefficients are background fields $\phi_{M_{1} \cdots M_{k}}(X)$ with indices that are contracted with the powers of $P^{M_{i}}$. Imposing the $\operatorname{Sp}(2, R)$ algebra under Poisson brackets requires the background fields to satisfy certain equations. The solution of these equations determines partially the form of the background fields, but still leaves undetermined functions that can be chosen according to physical considerations. As shown in [10], by keeping the lowest possible powers of $P$, one finds that all known fundamental interactions of particles (Yang-Mills, gravity, others) can be formulated generally in the $2 \mathrm{~T}$ formalism. The form of the $Q_{i j}$ is then

$$
\begin{aligned}
& Q_{11}=V^{M} V_{M}+\cdots, \quad Q_{12}=V^{M}\left(P_{M}+A_{M}\right)+\cdots \\
& Q_{22}=U+G^{M N}\left(P_{M}+A_{M}\right)\left(P_{N}+A_{N}\right)+\cdots
\end{aligned}
$$

where $A_{M}(X), G_{M N}(X), U(X)$ are the background fields for Yang-Mills, gravity and a scalar potential in $d+2$ dimensions. The dots $(\cdots)$ represent the terms with higher powers of $P$ including coefficients that represent higher spin fields. When all higher spin fields vanish one can show that the field $V^{M}(X)$ satisfies the coupled equations

$$
\begin{array}{ll}
V_{M}=\frac{1}{2} \partial_{M}\left(V^{K} V_{K}\right)=G_{M N} V^{N}, & V^{M} F_{M N}=0, \\
V^{M} \partial_{M} U=-2 U, & \mathfrak{£}_{V} G_{M N}=2 G_{M N},
\end{array}
$$

where $\mathfrak{f}_{V}$ is the Lie derivative with respect to $V$. These equations are generally covariant and Yang-Mills gauge invariant in $d+2$ dimensions.

After solving these equations explicitly, and imposing the constraints $Q_{11}=Q_{12}=0$ in a particular 1T gauge (going from $d+2$ to $d$ in a gauge similar to the free massless particle case of figure 1), it was shown in [10] that the $d+2$ equations of motion and the action (5) collapse to the system

$$
L=\frac{1}{2 A^{22}} \dot{x}^{\mu} \dot{x}^{v} g_{\mu \nu}(x)-\frac{A^{22}}{2} u(x)-\dot{x}^{\mu} A_{\mu}(x)
$$

which describes the motion of a relativistic spinless particle interacting with the arbitrary backgrounds $A_{\mu}, g_{\mu \nu}, u$ representing any gravitational, Yang-Mills or other interactions in $d$ dimensions.

The analysis may be repeated for other $1 \mathrm{~T}$ gauges (such as those related to figure 1) to establish relationships among interacting systems and unify them as a 2T-theory in a given background described by the action (5) in $d+2$ dimensions.

The analysis in [10] also included spinning particles by using $O S p(n \mid 2)$ instead of $\operatorname{Sp}(2, R)$. In this case there is a spin connection $\omega_{M}^{a b}(X)$ and a vector $V^{a}(X)$ where the index $a$ is a tangent space index in $d+2$ dimensions. Then one finds that the $O S p(n \mid 2)$ gauge 
symmetry of the action requires that the vector $V^{M}$, soldering form $E_{M}^{a}$, metric $G_{M N}$, torsion $T_{M N}^{a}=D_{[M} E_{N]}^{a}$, curvature $R=\mathrm{d} \omega+\omega^{2}$, are all constructed purely from $\omega_{M}^{a b}(X)$ and $V^{a}(X)$

$E_{M}^{a}=D_{M} V^{a}, \quad V^{M}=E_{a}^{M} V^{a}, \quad G_{M N}=E_{M}^{a} E_{N}^{b} \eta_{a b}, \quad T_{M N}^{a}=R_{M N}^{a b} V_{b}$,

where the covariant derivative $D_{M}$ uses the spin connection. The only restriction on $V^{a}$ and $\omega_{M}^{a b}$ is similar to the one satisfied by the Yang-Mills field

$$
V^{M} R_{M N}^{a b}=0, \quad V^{M} F_{M N}=0 .
$$

All the equations in (8) are solved by the definitions in (10) and the solutions ${ }^{6}$ of (11).

Thus, all fundamental interactions of particles for any spin in $d$ dimensions have a fully generally covariant and Yang-Mills gauge-invariant description in $d+2$ dimensions. As in the background-free case, many dynamical systems of an interacting particle in $d$ dimensions can be unified as a single 2T-theory in $d+2$ dimensions (different gauge choices to embed $d$ in $d+2$ ). The resulting classes of 1T-systems (similar to figure 1, but with background fields) have duality-type relations among them reflecting their common origin and hidden $(d+2)$-dimensional symmetries.

\section{2T field theory, unified 1T field theories}

$1 \mathrm{~T}$ field theory follows from imposing worldline theory constraints on the physical quantum states. Thus the constraint $p^{2}=0$ applied on states gives the Klein-Gordon equation $\partial_{\mu} \partial^{\mu} \phi=0$, which follows from the free-field Lagrangian $L=\frac{1}{2}\left(\partial_{\mu} \phi\right)^{2}$. Field interactions may then be added to consider an interacting field theory. We showed that one can derive many $1 \mathrm{~T}$ worldline theories from the same $2 \mathrm{~T}$ worldline theory by choosing gauges and solving explicitly the two constraints $X^{2}=X \cdot P=0$. The remaining constraint $P^{2}=0$ can take many different forms in terms of the remaining $d$ dimensions. For the relativistic massless particle in figure 1 it is $P^{2}=p^{2}=0$, while for the massive non-relativistic particle in figure 1 it is $P^{2}=-2 m H+p^{2}=0$, etc. Imposing $H=p^{2} / 2 m$ on the states gives the Schrödinger equation $\mathrm{i} \partial_{t} \psi=-\frac{1}{2 m} \nabla^{2} \psi$ that follows from the action $L=\mathrm{i} \psi^{*} \partial_{t} \psi-\frac{1}{2 m} \nabla \psi^{*} \cdot \nabla \psi$. Thus, various $1 \mathrm{~T}$ field theories follow from the same $2 \mathrm{~T}$ worldline theory when quantization is performed in different $1 \mathrm{~T}$ gauges. We will next show that we can derive the family of 1T field theories from the same $2 \mathrm{~T}$ field theory.

We quantize the worldline system covariantly in $d+2$ dimensions keeping the $S O(d, 2)$ symmetry manifest. This is done by imposing the $\operatorname{Sp}(2, R)$ constraints on the states to obtain the set of $\operatorname{Sp}(2, R)$ gauge-invariant physical states. However, since the constraints are non-Abelian we can diagonalize simultaneously only the Casimir and one of the $S p(2, R)$ generators, and then restrict to the singlet sector. When this procedure is applied [11] to the

6 Up to a general coordinate transformation one can choose a coordinate basis such that $X^{M}=\left(\kappa, \rho, x^{\mu}\right)$ while $V^{M}=(\kappa, \rho, 0)$. Then, up to a local $S O(d, 2)$ tangent space gauge transformation, and a Yang-Mills gauge transformation, the solution takes the following form:

$$
V^{a}=\kappa v^{a}, \quad \omega_{M}^{a b}=\left(\frac{1}{\kappa} u^{a b},-\frac{1}{\rho} u^{a b}, \omega_{\mu}^{a b}\right), \quad A_{M}=\left(\frac{1}{\kappa} a,-\frac{1}{\rho} a, A_{\mu}\right)
$$

where the functions $v^{a}, u^{a b}, \omega_{\mu}^{a b}, a, A_{\mu}$ are all arbitrary functions of $x^{\mu}$ and the ratio $\rho / \kappa$, while $E_{M}^{a}=$ $\left(\left(v^{a}+w^{a}\right),-\frac{\kappa}{\rho} w^{a}, \kappa e_{\mu}^{a}\right)$ with $e_{\mu}^{a}=D_{\mu} v^{a}$ and $w^{a}=\kappa \partial_{\kappa} v^{a}+u^{a b} v_{b}$. The $R_{\kappa \rho}^{a b}$ and $T_{\kappa \rho}^{a}$ components of curvature and torsion vanish in this coordinate basis, but the remaining components are generally non-zero. The general solution is valid before considering the constraints. The constraints $Q_{11}=Q_{12}=0$ are uniquely satisfied by setting $\rho=0=p_{\kappa}$. Then $e_{\mu}^{a}(x)$ together with $A_{\mu}(x)$ describe arbitrary gravitational and gauge interactions in the remaining $d$ dimensions $x^{\mu}$, including torsion. 
spinless particle system (3) the result is the manifestly $S O(d, 2)$ covariant field equations in $d+2$ dimensions,

$X^{2} \Phi(X)=0, \quad X^{M} \partial_{M} \Phi(X)=-\frac{d-2}{2} \Phi(X), \quad \partial^{2} \Phi(X)=g \Phi^{(d+2) /(d-2)}$.

The previous equation includes the only possible interaction consistent with the $\operatorname{Sp}(2, R)$ gauge singlet condition. The previous equation, which we call the 'dynamical equation' is derived from a Lagrangian

$$
L=-\frac{1}{2} \partial^{M} \Phi \partial_{M} \Phi-g \frac{d+2}{d-2} \Phi^{2 d /(d-2)}
$$

while the first two equations which we call the 'kinematic equations' are considered as subsidiary conditions which are not derived from this Lagrangian. Note that the kinematic equations correspond to the classical constraints $X^{2}, X \cdot P$. The equations in (13) at $g=0$ are identical to those derived by Dirac in 1936 with a rather different approach [21], with no knowledge of the underlying $\operatorname{Sp}(2, R)$ gauge symmetry. Now we see that his equations are simply the $S p(2, R)$ singlet condition on physical states.

Dirac showed that the four-dimensional Klein-Gordon equation $\partial_{\mu} \partial^{\mu} \phi=0$ follows from these $S O(4,2)$ covariant equations after solving the kinematic equations and coming down from six to four dimensions. He thus proved that the conformal symmetry $S O(4,2)$ of the massless Klein-Gordon equation becomes manifest in the form of (13).

However, Dirac and his followers who were not aware of the underlying $\operatorname{Sp}(2, R)$ gauge symmetry, did not notice that it is possible to come down from six to four dimensions in very different ways by choosing different $1 \mathrm{~T}$ gauges. The form of the 1T field theory depends on the embedding of $d$ dimensions in $d+2$ dimensions and solving the kinematic equations by using those coordinates. Replacing the solution in the previous equation in (13) yields the $1 \mathrm{~T}$ dynamical equation in $d$ dimensions. Furthermore, the 2T Lagrangian (14) reduces to the correct $1 \mathrm{~T}$ field theory Lagrangian. Using this procedure, it was shown in [11] that the Schrödinger equation and other equations that correspond to the first quantized field equations for the systems in figure 1 follow from (13). Therefore, the same 2T field theory in $d+2$ dimensions unifies many types of $1 \mathrm{~T}$ field theories in $d$ dimensions, such as those that correspond to figure 1 . The local field interactions in $d+2$, as in (14), result in various forms of interaction in different $1 \mathrm{~T}$ gauges. The interaction has the same form as (14) in the gauge that corresponds to the relativistic massless particle in figure 1, but can be more involved in other gauges (for examples see [11]).

A similar approach works for spinning particles. The $S O(d, 2)$ covariant quantization of the $2 \mathrm{~T}$ worldline theory leads to a formulation of $2 \mathrm{~T}$ interacting classical field theory for spinning fields. In $d+2$ dimensions spinors have twice as many components as in $d$ dimensions, so how can the $d+2$ field theory become the same as the $d$-dimensional field theory? Interestingly, the $O S p(1 \mid 2)$ gauge singlet condition produces a new type of spinor field equation (and Lagrangian) which have a kappa-type symmetry that cuts down the number of spinor components by a factor of two. The process also generates the Yang-Mills and gravitational equations with consistent interactions in $d+2$ dimensions. One finds that the equations in (11) together with $X^{2}=0$ are the kinematical equations for the Yang-Mills and gravitational fields, while the dynamical equations, including interactions, follow from a Lagrangian as given in [11]. Then one sees that the standard model may be regarded as a 2T field theory in six dimensions which is reduced to four dimensions in a particular gauge.

Our current understanding of the $2 \mathrm{~T}$ field theory formulation is somewhat incomplete. This is because the kinematic equations such as those in (13) and (11) are imposed as additional field equations which do not follow from the field theory Lagrangian. They correspond to the 
worldline theory constraints $Q_{11}=Q_{12}=0$. A more satisfactory situation would be to derive all the equations, not only the dynamical equation, directly in the field theory formalism. One approach is to introduce Lagrange multipliers, but this seems artificial. A more natural 2T field theory may need to be based on fields that depend on phase space $\phi(X, P)$ and which transform under a local $S p(2, R)$ symmetry (this is somewhat reminiscent of non-commutative geometry). Then the $\operatorname{Sp}(2, R)$ singlet equations (both kinematic and dynamic) could follow from the gauge symmetry of the field theory rather than the gauge symmetry of the worldline theory. This formulation remains as a challenge.

Although the $2 \mathrm{~T}$ unification of 1T-systems can be examined in either the worldline or field theory forms, the worldline approach provides a better understanding of the underlying gauge symmetries at this stage, while the field theory formulation provides an approach for interactions among fields.

Second quantization of 2T-physics may be considered. The improvement of the field theory mentioned above would probably help in the formulation of the second quantization.

\section{Local and global $2 \mathrm{~T}$ supersymmetry}

The generalization of the 2T-theory to spacetime supersymmetry emerged in several steps by developing a new approach to both global and local kappa supersymmetries [5-9]. The generalized form of (3) that applies in several situations of physical interest is

$$
S=\int \mathrm{d} \tau\left[\left(\dot{X}_{1}^{M} X_{2}^{N}-\frac{1}{2} A^{i j} X_{i}^{M} X_{j}^{N}\right) \eta_{M N}-\operatorname{Str}\left(L\left(\partial_{\tau} g g^{-1}\right)\right)\right],
$$

where $g \in G$ is a supergroup element, and $L=L^{M N} \Gamma_{M N}$ is a coupling of the Cartan form $\partial_{\tau} g g^{-1}$ to the orbital $S O(d, 2)$ Lorentz generators $L^{M N}=\varepsilon^{i j} X_{i}^{M} X_{j}^{N}$. The supergroup element $g$ contains fermions $\Theta$ that are now coupled to the $X_{i}^{M}$.

Let us define $G_{L}$ as the supergroup under left multiplication $g \leftarrow g_{L} g$. We require that the bosonic subgroup of $G_{L}$ contain at least $S O(d-k, 2) \times S O(k)$ for some $k=0, \ldots, d-2$. This is identified with part of the Lorentz group $S O(d, 2)$ that rotates the $X_{i}^{M}$. The full bosonic subgroup of $G_{L}$ may contain an additional factor $h_{L}$. The matrices $\Gamma_{M N}$ (up to fixed coupling coefficients that depend only on $d, k)$ represent $S O(d-k, 2) \times S O(k)$ and provide the coupling of $L^{M N}$ to the appropriate part of the Cartan connection. If there is an additional bosonic subgroup $h_{L}$ then the $L^{M N}$ have zero coupling to the corresponding part of the Cartan form.

For the right choices of $G$ the fermions $\Theta$ transform as a spinor at least under $S O(d-k, 2)$ (and either spinor or other representation under $S O(k) \times h_{L}$ ). Then they have the correct spacetime spinor properties to become the supercoordinates for a spacetime with several supersymmetries. The structure of the Lagrangian above guarantees the following local and global symmetries.

- There is a local $\operatorname{Sp}(2, R)$ symmetry. The first term in (15) is invariant just like (3). The second term is invariant because $L^{M N}$ is invariant and $g$ is a singlet under $\operatorname{Sp}(2, R)$.

- There is local symmetry under the bosonic subgroup $S O(d-k, 2) \times S O(k) \times h_{L} \in G_{L}$. The $S O(d-k, 2) \times S O(k)$ part also transforms $X_{i}^{M}$ locally. This can be used to eliminate part or all of the bosonic degrees of freedom in $g$, or part of the $X_{i}^{M}$. In this way one may rewrite the theory either in terms of spacetime vectors or spacetime twistors (see [7] for an example).

- There is a kappa-type local supersymmetry under part or all of the fermionic transformations in $G_{L}$. The local parameters kappa are isomorphic to $\Theta$ with some 
projectors applied to it. The projectors take into account the constraints $X_{i} \cdot X_{j} \sim 0$ that arise because of the local $S p(2, R)$. Because of the projectors kappa supersymmetry can remove part but not all of the $\Theta(\tau)$ degrees of freedom. The fraction of $\Theta$ that can be removed ranges from 0 to $\frac{3}{4}$ depending on the specific details of the couplings in (15).

- There is a global supersymmetry $G_{R}$ under right multiplication of $g \rightarrow g g_{R}$. The $X_{i}^{M}$ do not transform when all gauge degrees of freedom in $g$ are included. This is the global spacetime supersymmetry $G_{R}$. It mixes only the bosons and fermions in $g$, and it is realized linearly on them. Although the $X_{i}^{M}$ do not transform in this version, they do transform after eliminating some bosons from $g$ by gauge fixing the $S O(d-k, 2) \times S O(k) \times h_{L}$ gauge symmetries. When $g$ is gauge fixed, both $G_{L}$ and $G_{R}$ must act on it to maintain the gauge. Then the global $G_{R}$ transformation is induced on the $X_{i}^{M}$ as a field-dependent (including $\Theta$-dependent) local $S O(d-k, 2) \times S O(k)$ transformation. The resulting transformation becomes precisely the needed global spacetime supersymmetry in $d+2$ dimensions. In this version, $G_{R}$ mixes $\Theta, X_{i}^{M}$ (and any bosons in $g$ that may have remained after fixing the $G_{L}$ gauge symmetries). When the 2T-theory is further fixed to some kappa gauge and some 1T $\operatorname{Sp}(2, R)$ gauge, the $d+2$ supersymmetry (that has twice as many fermionic parameters) reduces to the correct spacetime supersymmetry in $d$ dimensions. It must be emphasized that since there are many possible $1 \mathrm{~T}$ gauges as in figure 1, the resulting supersymmetry is the correct one for those $1 \mathrm{~T}$ theories.

A few applications of the general scheme are given in the next three subsections.

\subsection{Superparticle in $d=3,4,6$}

The simplest example of a supersymmetric action was discussed in [5,7] for $d=3,4,6$ dimensions with $N$ supersymmetries. For these dimensions we take $G=O S p(N \mid 4)$, $S U(2,2 \mid N), O S p\left(8^{*} \mid N\right)$, respectively. These supergroups contain the $S O(d, 2)$ spacetime subgroups $S O(3,2)=S p(4), S O(4,2)=S U(2,2), S O(6,2)=\operatorname{Spin}\left(8^{*}\right)$, respectively, to which $L$ is coupled (i.e. $k=0)$. There is zero coupling to the internal subgroups $h_{L}=S O(N)$, $S U(N), S p(N)$, respectively. The fermionic parameters or supercharges are classified as $(4, N),(4, N)+(\overline{4}, \bar{N}),\left(8^{*}, N\right)$, respectively, where the $4,4+\overline{4}, 8^{*}$ correspond to the spinor representations of the corresponding $S O(d, 2)$. When all the gauge fixing is done, in the $1 \mathrm{~T}$ gauge that corresponds to the massless particle of figure 1, the action (15) reduces to the following form in $d$-dimensional superspace $\left(x^{\mu}, \theta_{\alpha}^{a}\right)$ with $N$ supersymmetries $a=1,2, \ldots N$

$$
£=\dot{x} \cdot p-\frac{1}{2} A^{22} p^{2}+\tilde{\theta}_{a} \gamma \cdot p \partial_{\tau} \theta^{a} \Rightarrow \frac{1}{2 A^{22}}\left(\dot{x}^{\mu}+\tilde{\theta}_{a} \gamma^{\mu} \partial_{\tau} \theta^{a}\right)^{2} .
$$

This is the well known action for the massless superparticle. In the special dimensions $d=3,4,6$ it does indeed have the larger global supergroup symmetry $G=O S p(N \mid 4)$, $S U(2,2 \mid N), O S p\left(8^{*} \mid N\right)$, respectively, that includes the additional $N$ hidden superconformal symmetries [5,29].

It is important to emphasize again that one could choose gauges that correspond to other $1 \mathrm{~T}$ particle systems such as those of figure 1 . One would then find the supersymmetrized versions for all of them in the special dimensions $d=3,4,6$. These are new supersymmetric actions that were not noticed before except for the case of the supersymmetric $\mathrm{H}$-atom in $d=4$ [30], but even then the larger hidden symmetries were not known.

\section{2. $A d S_{5} \times S^{5}$ supersymmetric Kaluza-Klein tower}

In the previous paragraph the spacetime and internal subgroups of $O S p(8 \mid 4), S U(2,2 \mid 4)$, $O S p\left(8^{*} \mid 4\right)$ were treated in an asymmetric manner. The result was a superparticle moving in 
flat space in $d=3,4,6$ only. Since these supergroups describe the supersymmetries in the curved spaces $A d S_{7} \times S_{4}, A d S_{5} \times S_{5}, A d S_{4} \times S_{7}$, respectively, one may wonder whether there is a more interesting treatment of the subgroups that would apply to these cases.

We describe here the $A d S_{5} \times S_{5}$ case discussed in [8]. We take $g \in S U(2,2 \mid 4)$ and 12 dimensions $X^{M}, P^{M}$ with signature $(10,2)$. We divide them into two sets of six dimensions each, $X^{m}, P^{m}$ with signature $(4,2)$ and $X^{a}, P^{a}$ with signature $(6,0)$. The $S O(10,2)$ orbital angular momentum now has components $L^{m n}, L^{m a}, L^{a b}$. In the coupling scheme only $L^{m n} \sim S U(2,2)$, and $L^{a b} \sim S U(4)$ are coupled in the action (15), while $h_{L}=0$ does not exist. The kappa supersymmetry has a parameter of the form $\xi=L^{m a}\left(\Gamma_{m} \kappa \Gamma_{a}\right)$ where $\xi$ is an infinitesimal group parameter in $G_{L}$ classified as $(4, \overline{4})$ under $S U(2,2) \times S U(4) \subset G_{L}$. The coset orbital angular momentum $L^{m a}=X^{m} P^{a}-X^{a} P^{m}$ plays the role of a projector applied on the free local fermionic parameter $\kappa(\tau)$, which is also classified as $(4, \overline{4})$. The kappa transformation (a fermionic $G_{L}$ transformation applied only $g$ and on $A^{i j}$ ) gives $\delta £=\left(\delta A^{i j}+K^{i j}\right) X_{i} \cdot X_{j}$, where $K^{i j}$ comes from the second term in the action; it is a complicated expression that depends on $g, X_{i}^{M}, \kappa$. Note the important fact that $K^{i j}$ multiplies $X_{i} \cdot X_{j}$ involving both $X_{i}^{m}$ and $X_{i}^{a}$ in the precise combination that corresponds to the constraint $X_{i} \cdot X_{j}$. Then we can choose $\delta A^{i j}$ so that $\delta A^{i j}+K^{i j}=0$ to have the kappa symmetry, $\delta £=0$.

A possible gauge choice is the following. Using the $\operatorname{Sp}(2, R)$ local symmetry we can choose two gauges: the component $P^{+\prime}$ of the $S O(4,2)$ vector $X^{m}$ vanishes for all $\tau, P^{+\prime}=0$, and the magnitude of the $S O(6)$ vector is a $\tau$-independent constant $\left|X^{a}\right|=a$. Thus the $A d S_{5} \times S^{5}$ curved background is created from the flat 12-dimensional background with this gauge choice (for details see [2]). Furthermore, using the local $G_{L}$ symmetry we can eliminate all the bosons and half of the fermions from $g$. Then $g$ contains only eight complex fermions or 16 real fermions that are nonlinearly coupled to the orbital $A d S_{5} \times S^{5}$ symmetry operators $L^{m n}, L^{a b}$ (which themselves are now nonlinearly realized as given in [2]).

The gauge-fixed action describes the entire $A d S_{5} \times S^{5}$ supersymmetric Kaluza-Klein tower [8] which was previously derived in the context of compactified 10D type IIB supergravity. Note the following simple facts. First, the 16 fermions provide Ramond-type vacua with 128 bosons and 128 fermions. These correspond just to the fields of supergravity that can be reclassified under the global symmetry group $S U(2,2 \mid 4)$. They are now propagating in the $A d S_{5} \times S^{5}$ background. Second, although the constraints $X^{2}=X \cdot P=0$ have been solved explicitly in the chosen $\operatorname{Sp}(2, R)$ gauge, the remaining 12-dimensional constraint $P^{2}=0$ takes the following sketchy form:

$$
\text { Casimir of } S O(4,2)+\text { Casimir of } S O(6)=0 \text {. }
$$

Thus, the mass of the field as given by the Casimir of $S O(4,2)$ is now fully determined by the Casimir of $S O(6)$. The Casimir of $S O(6)$ for the graviton tower is determined by the $S O(6)$ representations that can be constructed from the traceless symmetrized products of $X^{a}$. The traceless tensor with $l$ indices has the $S O(6)$ Casimir eigenvalue $l(l+4)$. The mass of the rest of the supermultiplet is determined by the $S U(2,2 \mid 4)$ supersymmetry, which is built in from the beginning.

\subsection{Toy M-model}

The toy 'M-model' sketched here was introduced in [6] and analysed in [9]. It illustrates some of the general aspects of M-theory in the context of 2T-physics that is explained in the next section. Consider the Lagrangian given by (15) with $d+2=13$, and $g \in O S p(1 \mid 64)$. The coupling $L$ is to the $S O(11,2)$ subgroup of $O S p(1 \mid 64)$. It can be shown that in one gauge (the one closest to the massless particle in figure 1) the model describes $d=11$ 
particle super-coordinates $x^{\mu}, \theta$, together with collective coordinates $x_{\mu \nu}, x_{\mu_{1} \cdots \mu_{5}}$ for the 11D 2-brane and 5-brane. In this gauge the linearly realized part of $O S p(1 \mid 64)$ is precisely the 11D extended superalgebra of equation (1) with certain constraints among the commuting brane charges. The model has enough gauge symmetries (including bosonic extensions of kappatype supersymmetries) to remove all ghosts associated with all 11D-covariant super degrees of freedom that occur in the 2-brane and 5-brane collective degrees of freedom [9]. The quantum toy 'M-model' is unitary. Furthermore, in this gauge all degrees of freedom can be recast to the language of super-twistors (using the techniques of [7]). The resulting formalism is similar to the super-oscillators as in [28] with 16- 'colours', and we must also require 'colour'singlet physical states. The physical spectrum can then be easily determined: 128-bosons and 128-fermions (states of 11D supergravity) in the presence of 2- and 5-brane charges with certain relations among them. The model in this gauge realizes a BPS representation (a short representation) of $O S p(1 \mid 64)$, with 32 vanishing supercharges. The other non-vanishing 32 supercharges correspond to 16 linearly realized supersymmetries plus 16 nonlinearly realized superconformal symmetries.

\section{M-theory and 2T}

Since we have demonstrated that well known physics, including the standard model, gravity and possibly string theory (see the next section), have a 2T-physics formulation which sheds a deeper light into symmetries and higher dimensions, we are encouraged to apply the same concepts to M-theory. After all, 2T-physics started with hints in the mathematical structure of M-theory, F-theory and S-theory as described in the introduction. In the following we will refer to the underlying theory as 'M-theory', but it will be evident that the remarks can apply to M-theory, F-theory or S-theory seen as corners of the underlying 'M-theory'.

For 'M-theory' 2T-physics makes a general prediction independent of any details [6]. It requires that the explicit and hidden global symmetries of 'M-theory' must be described by the non-Abelian $O S p(1 \mid 64)$ superalgebra. This should be useful for the eventual formulation of the fundamental theory. The reasoning is simple. It is likely that hidden spacetime symmetries of 'M-theory' would be displayed by adding $(1,1)$ dimensions to the 11 dimensions already noticed, with signature $(10,1)$. Then the 2T-physics version of 'M-theory' would be based on 13 dimensions with $(11,2)$ signature. If one adds supersymmetry and requires $S O(11,2)$ covariance, one must have $S O(11,2)$ spinors that have 64 components. The unique closure of the 64 supercharges that contains $S O(11,2)$ is $O S p(1 \mid 64)$. This then must be the global supersymmetry of 'M-theory'. Note that we insist on the non-Abelian supergroup, not a contracted version. As shown in [6], the usual 11D superalgebra (1) with commuting momenta and commuting 2- and 5-brane charges is part of the non-Abelian $O S p(1 \mid 64)$. The Abelian 11D M-superalgebra has a 'triangular' type of embedding in the non-Abelian 13D superalgebra.

The 2T 'M-theory' must also have a gauge symmetry analogous to $\operatorname{Sp}(2, R)$ (or its generalizations discussed in the previous sections). Although the gauge symmetry is unknown at this time, its presence would eliminate ghosts, on the one hand, and provide 1T 'holographic' pictures of the $13 \mathrm{D}$ theory in lower dimensions, on the other hand. Then we may view various corners of M-theory as gauge-fixed versions of the $2 \mathrm{~T}$ formulation to various $1 \mathrm{~T}$ formulations. In that case we would expect that every $1 \mathrm{~T}$ version should have the $O S p(1 \mid 64)$ symmetry, with part of it linearly realized and the remainder nonlinearly realized and somewhat hidden.

Further support for this point of view can be found in the fact that $O S p(1 \mid 64)$ correctly contains the various supersymmetries of several corners of S-theory, F-theory and M-theory, including 13D, 12D, 11D, 10D type-IIA, 10D type-IIB, heterotic, type-I, and $A d S \times S$ supersymmetries. This has been demonstrated in [6]. The relevant non- 
Abelian $A d S \times S$ superalgebras can be fitted in $O S p(1 \mid 64)$ only after some contractions which could be associated with large $N$ limits that occur in discussions involving the AdSCFT correspondence. The uncontracted versions of the relevant $A d S \times S$ supersymmetries contain additional brane charges such that the closure is uniquely $O S p(1 \mid 32)$ contained in $O S p(1 \mid 64)[6,31]$. One can also include $O S p\left(8^{*} \mid 8\right)$ discussed recently [32] in the list of interesting algebras contained in $O S p(1 \mid 64)$ (with some contraction) as being relevant for describing some corners of 'M-theory'.

\section{Strings and branes}

For a $p$-brane $X^{M}\left(\tau, \sigma_{1}, \ldots, \sigma_{p}\right)$ the natural candidate for a local symmetry that replaces $S p(2, R)$ is the conformal group on the worldvolume $S O(p+1,2)$. This was pointed out in [1]. Localizing this group implies coupling $X^{M}(\tau, \vec{\sigma})$ to the gauge fields of conformal gravity on the brane. In particular, for $p=0$ this yields the same Lagrangian as local $\operatorname{Sp}(2, R)=\operatorname{SO}(1,2)$ in the second-order formalism obtained by integrating out $P^{M}$ in equation (3). A first-order formulation of this theory analogous to the $\operatorname{Sp}(2, R)$ formulation has been constructed [33]. The global symmetry is again $S O(d, 2)$ in flat space. The gauge symmetry and constraints are such that $d+2$ is reduced to $d$ dimensions for the entire brane for any $p$. A (partial?) formulation of 2T-physics for $p$-branes based on this idea has been published in [4], although in that paper only a related and simplified version of the formalism was analysed which appeared useful at the time. It was shown that $1 \mathrm{~T}$ tensionless and rigid branes on flat or $A d S \times S$ backgrounds could be obtained from the $2 \mathrm{~T} p$-brane by choosing certain $1 \mathrm{~T}$ gauges for $S O(p+1,2)$. A gauge that describes branes with tension (such as massive particles of figure 1) seemed difficult to find. It was not understood whether the search for gauge choices was not sufficiently broad or whether the Lagrangian could be missing some terms. Thus, more work needs to be done to establish the $2 \mathrm{~T}$ formalism more generally for $p$-branes.

\section{Outlook}

The 2T point of view of ordinary 1T-physics has revealed a new subtle dimensional unification in $d+2$ dimensions. The $2 \mathrm{~T}$ language is the only explanation of the symmetries and the relations among the 1T dynamical systems in the same family. Furthermore, the action looks simpler, more elegant, more symmetric and displays a fundamental gauge principle which is not apparent in 1T-physics.

The gauge symmetry is similar to 'duality' if analysed from the point of view of 1Tsystems. Each 1T-system can be considered as a $d$-dimensional 'holographic' image of the $d+2$ theory. Only, holography here gives an image projected on a 'surface' of two fewer spacetime dimensions. Each 1T holographic image appears as a different dynamical system from the point of view of 1T-physics. The global symmetry $S O(d, 2)$ is observable in every holographic image. It is one of the key evidences for 2T-physics which could be verified experimentally or computationally either within a given $1 \mathrm{~T}$-system (e.g. H-atom as an $S O(4,2)$ system, etc) or in duality relations among several systems as outlined in a previous section. In principle, there are an infinite number of such duality relations, and it would be an interesting project to compile some predictions that could be tested experimentally or computationally when such computations are possible.

The known physics of the standard model has a $2 \mathrm{~T}$ field theory description. It appears as a four-dimensional holographic image of a 2T-theory in $4+2$ dimensions. The general $2 \mathrm{~T}$ field theory formulation can probably be improved and put into a more fundamental form so 
that all equations (kinematic + dynamic) follow from the same field theory. This more general formulation may involve aspects of non-commutative geometry.

Based on the insights brought by 2T-physics into 1T-physics, it is reasonable to expect that 'M-theory' has sufficient hidden symmetries that amount to as much as a non-Abelian $O S p(1 \mid 64)$. It may be helpful to use this remark as a guide to build more systematics about 'M-theory' and to come closer to an eventual construction of the theory.

There are many solveable problems in furthering the techniques of 2T-physics, using it in new physical applications or computations involving ordinary macroscopic or microscopic physics, and finding experimental tests to verify some of its predictions. There are also some technical and conceptual projects that include the following.

- Completion of the 2T formalism for strings and branes.

- Analysis of various aspects of 2T field theory, including relations among $1 \mathrm{~T}$ field theories, in particular those related to QCD or the standard model. Can we learn something new and non-perturbative about QCD or the standard model in this process?

- Backgrounds in the presence of supersymmetry. This is expected to lead to supersymmetric Yang-Mills and supergravity in $d+2$ dimensions. In particular, we know that backgrounds for the 10D superparticle must obey the super-Yang-Mills equations to preserve the kappa supersymmetry. The $2 \mathrm{~T}$ formulation of this system must then yield $(10,2)$ super-Yang-Mills including the dynamical equations, not only the kinematic ones. The same is expected for supergravity in $(10,2)$ or $(11,2)$ dimensions. These are theories that have been sought for but never constructed satisfactorily before. The $2 \mathrm{~T}$ approach might do it.

- There is renewed interest in higher spin fields. These have been mainly analysed in $d=3$ dimensions based on the conformal group $S O(3,2)=S p(4)[34,35]$. The background field scheme involving higher powers of $P^{M}$ described in a previous section provides a new formalism for discovering the properties and the field equations for higher spin fields in an explicitly $S O(d, 2)$ covariant approach in any dimension.

- Construction of 'M-theory'.

What message is there about spacetime? Are there really two times, are there really 13 dimensions? The formalism of 2T-physics teaches us that one extra spacelike and one extra timelike dimensions do exist, but not in the naive sense. The usual four (or $d$ ) dimensions together with the extra two dimensions define a particular 'holographic' view of a 2T system in a spacetime with six (or $d+2$ ) dimensions. This 'holographic' picture appears as a dynamical system described by 1T-physics in four (or $d$ ) dimensions. A different $1 \mathrm{~T}$ dynamical system is obtained from a different 'holographic' view of the same $2 \mathrm{~T}$ system by embedding four (or $d$ ) plus tow dimensions differently in six $($ or $d+2)$ dimensions. To obtain all possible 'holographic' views, corresponding to all possible 1T dynamical systems in the same family, all the $d+2$ dimensions are needed on an equivalent basis. In this sense all the $d+2$ dimensions exist. They reveal themselves in any of the holographic pictures described by 1T-physics by the presence of hidden symmetries and in the relations among many 1T-systems. 2T-physics teaches us that spacetime can be more than what we used to think of before.

\section{Acknowledgments}

This research was partially supported by the US Department of Energy under grant number DE-FG03-84ER40168. The paper is based on lectures delivered at the Workshop on Strings, Branes and M-theory, CIT-USC Center, Los Angeles (March 2000); Second International School on Field Theory and Gravitation, Vitória, Brazil (April 2000); Quantization Gauge 
Theory and Strings, Moscow (June 2000); Non-perturbative Methods in Field and String Theory, Copenhagen (June 2000); M-theory and Dualities, Istanbul (June 2000).

\section{References}

[1] Bars I, Deliduman C and Andreev O 1998 Phys. Rev. D 58066004

(Bars I, Deliduman C and Andreev O 1998 Preprint hep-th/9803188)

Bars I 1998 Phys. Rev. D 58066006

(Bars I 1998 Preprint hep-th/9804028)

Vongehr S 1999 Examples of black holes in two time physics Preprint hep-th/9907077

For a review, see Bars I 1998 Preprint hep-th/9809034

[2] Bars I 1999 Phys. Rev. D 59045019

(Bars I 1998 Preprint hep-th/9810025)

[3] Bars I and Deliduman C 1998 Phys. Rev. D 58106004 (Bars I and Deliduman C 1998 Preprint hep-th/9806085)

[4] Bars I, Deliduman C and Minic D 1999 Phys. Lett. B 466135 (Bars I, Deliduman C and Minic D 1999 Preprint hep-th/9906223)

[5] Bars I, Deliduman C and Minic D 1999 Phys. Rev. D 59125004 (Bars I, Deliduman C and Minic D 1998 Preprint hep-th/9812161)

[6] Bars I, Deliduman C and Minic D 1999 Phys. Lett. B 457275 (Bars I, Deliduman C and Minic D 1999 Preprint hep-th/9904063)

[7] Bars I $20002 \mathrm{~T}$ formulation of superconformal dynamics relating to twistors and supertwistors Preprint hepth/0004090

[8] Bars I 2001 The $\mathrm{AdS}_{5} \times \mathrm{S}^{5}$ super Kaluza-Klein Tower as a worldline 2T-theory, in preparation

[9] Bars I 2001 A toy M-model, in preparation

[10] Bars I 2000 Two-time physics with gravitational and gauge field backgrounds Preprint hep-th/0002140

[11] Bars I 2000 Two-time physics in field theory Preprint hep-th/0003100

[12] Townsend P 1995 p-brane democracy Preprint hep-th/9507048

[13] Bars I 1995 Duality and hidden dimensions Proc. Conf. Frontiers in Quantum Field Theory (Toyonaka, 1995) ed Itoyama et al p 52

(Bars I 1996 Preprint hep-th/9604200)

Bars I 1996 Phys. Rev. D 545203

[14] Bars I 1997 Phys. Rev. D 552373

Bars I 1996 Algebraic structures in S-theory Preprint hep-th/9608061 (Lectures in 2nd Sakharov Conf. 1996, Strings-96 Conf.)

Bars I 1997 Phys. Lett. B $\mathbf{4 0 3} 257$

[15] Duff M and Blencowe M P 1988 Nucl. Phys. B 310387

[16] Ooguri H and Vafa C 1990 Mod. Phys. Lett. A 51389

Ooguri H and Vafa C 1991 Nucl. Phys. B 361469

Ooguri H and Vafa C 1991 Nucl. Phys. B 36783

[17] Vafa C 1996 Nucl. Phys. B 469403

[18] Hull C M

[19] Nishino H and Sezgin E 1996 Phys. Lett. B 388569

Sezgin E 1997 Phys. Lett. B 403265

Nishino H 1997 Preprint hep-th/9710141

[20] Maldacena J 1998 Adv. Theor. Math. Phys. 2231

(Maldacena J 1997 Preprint hep-th/9711200)

Maldacena J 1999 Int. J. Theor. Phys. 381113

[21] Dirac P A M 1936 Ann. Math. 37429

[22] Kastrup H A 1966 Phys. Rev. 1501183

[23] Mack G and Salam A 1969 Ann. Phys. 53174

[24] Preitschopf C R and Vasilev M A 1998 Preprint hep-th/9812113

[25] Marnelius R 1979 Phys. Rev. D 202091

Nilsson B and Marnelius R 1980 Phys. Rev. D 22830

Siegel 1988 Int. J. Mod. Phys. A 32713

[26] Bars I 2001 Multi-particles with multi-times, in preparation

[27] Bars I and Kounnas C 1997 Phys. Lett. B 40225 
Bars I and Kounnas C 1997 Phys. Rev. D 563664

Bars I and Deliduman C 1997 Phys. Rev. D 566579

(Bars I and Deliduman C 1997 Preprint hep-th/9707215)

Bars I and Deliduman C 1998 Phys. Lett. B 41724

(Bars I and Deliduman C 1997 Preprint hep-th/9710066)

Sezgin E 1997 Phys. Lett. B 403265

Sezgin E and Rudychev 1997 Preprint hep-th/9711128

Sezgin E and Rudychev 1998 Phys. Lett. B 42460

[28] Bars I and Günaydin M 1983 Commun. Math. Phys. 9131

Günaydin M and Marcus N 1985 Class. Quantum Grav. 2 L11

Günaydin M, Minic D and Zagermann M 1998 Nucl. Phys. B 53496

Günaydin M, Minic D and Zagermann M 1999 Nucl. Phys. B 544737

Günaydin M and Minic D 1998 Nucl. Phys. B 523145

[29] Schwarz J 1981 Nucl. Phys. B 185221

[30] d'Hoker E and Vinet L 1985 Nucl. Phys. B 26079

[31] Ferrara S and Porrati M 1999 Preprint hep-th/9903241

[32] Hull C M 2000 Strongly coupled gravity and duality Nucl. Phys. B $\mathbf{5 8 3} 237$

[33] Bars I 2001 2T Strings and branes, in preparation

[34] Prokushkin S and Vasiliev M 1999 Nucl. Phys. B 545385 and references therein

[35] Sezgin E and Sundell P 1999 Higher spin $n=8$ supergravity in ads(4) Preprint hep-th/9903020 